\title{
Traffic Controller Based on Density with RF Remote Override
}

\author{
P V Ramaraju, G. Nagaraju, V.N.Ganeswarreddy, V.Sai Kanna, B.Sashank
}

\begin{abstract}
The endeavor hopes to give a sensible response for the traffic signal structure to deny the standard sign timings during emergency regularly. It happens when there is an emergency condition like crisis vehicle, fire division stuck in flood hour gridlock; they imagine that need should go first. Other than the need rises when there develops high thickness at a particular course. Thusly the system uses an android application device remote control that invalidations the sign timings by essentially offering green piece of information in the vehicle course and red sign for all others. The endeavor uses a microcontroller of 8051 family that is interfaced with the IR sensors and photodiodes balanced in discernable pathway diagram over the store for seeing the thickness. The thickness is surveyed in three stand-out ways low, medium and high as appeared by which the timings are appropriated for sign. The managing supplanted is done using RF advance.
\end{abstract}

Keywords:Traffic signals, Micro controller, IR Sensors, PCB, Remote override

\section{INTRODUCTION}

In the present energetic life, traffic stop up changes into an essential issue in our standard activities. It hacks down the effectiveness of individual and everything considered the general masses as heaps of work hour is wasted in the sign. High volume of vehicles, the lacking system and the astonishing scattering of the hailing structure are standard purposes behind this disarranged blockages. It in a smart manner in like manner adds to the improvement in sullying level as engines remain on all around, a tremendous volume of trademark resources in sorts of oil and diesel is used with no productive outcome. In like manner, to discard these issues or perhaps rot them to crucial level, consistently current plans ought to be executed by getting sensor based computerization strategy in this field of traffic hailing structure

\section{PRESENT TRAFFIC SIGNALING SYSTEM:}

Under current condition, traffic control is cleaned by the use of a system of hand signs by traffic police work control, traffic sign, and markings. An in each prudent sense foggy and arranging preparing try is required, through driverfavoring specialists, to ensure that the thorough system who

Revised Manuscript Received on April 12, 2019.

P V Ramaraju, Professor, Department of ECE, SRKR Engineering College, Bhimavaram, A.P, India..(pvrraju50@gmail.com)

G. Nagaraju, Asst. Professor, Department of ECE, SRKR Engineering College, Bhimavaram, A.P, India.. (bhanu.raj.nikhil@gmail.com)

V.N.Ganeswarreddy, B.Tech Student, Department of ECE, SRKR Engineering College, Bhimavaram, A.P, India. (ganeshreddy143341@gmail.com)

V.SaiKanna, B.Tech Student, Department of ECE, SRKR Engineering College, Bhimavaram, A.P, India.. (saikanna2016@gmail.com)

B.Sashank,B.Tech Student, Department of ECE, SRKR Engineering College, Bhimavaram, A.P, India.. (sashankbommadevara@gmail.com) work motor vehicles value the benchmarks of the road and the exercises that they are required or referenced to take when a particular control contraption is open. Each traffic control contraption is obliged by degrees of structure and use; for example, stop signs dependably have a red establishment and are octagonal fit as a fiddle. Plan models interface with the driver to quickly andconsistentlyperceivethesignin the visual field along the road. Standard use of tints and shape helps in this specific assertion and in decidingon the correct course of action. Under current conditions, trafficlightsareset on in the different ways with fixed time delay, following a particular cycle while changing from one sign to other making unwantedand wasteful congestionon one way while various ways remain void. The system we propose see the thickness of traffic on individual ways and therebyregulate the arranging of the sign's sorting out. IR trans beneficiaries check the snags and give an idea concerning the traffic thickness on a particular way and feed this response to a controller unit which will pick the fundamental decisions as and when require.

\section{OPERATIONAL MODEL:}

This endeavor goes for decreasing traffic stop up and horrendous time delay during the traffic light switch overs especially when the traffic is incredibly low. It is relied upon to execute in spots near the mixes, where the traffic sign are set in, to lessen the stop up in flood hour gridlock. It screens number of vehicles on each side of crossing point and correspondingly changes the perfect open entryway for each traffic light standard. The higher the degree of vehicles out on the town the more drawn out will be the time give up dissipated for that disconnecting traffic light sign.

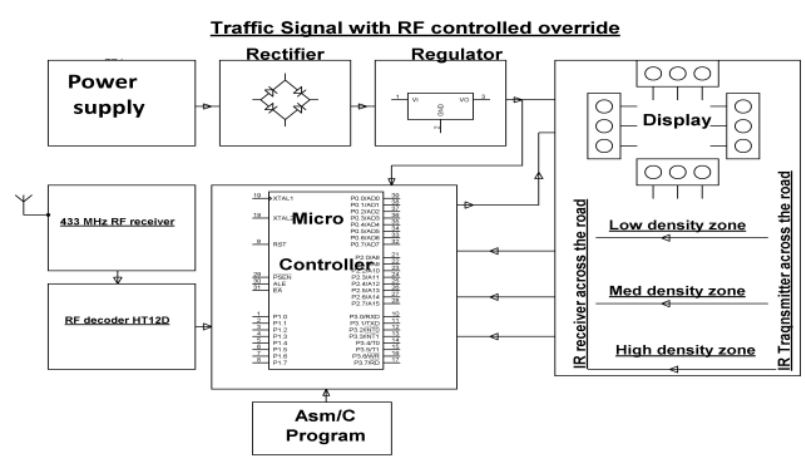

Fig: Basic Block Diagram

Published By:

Blue Eyes Intelligence Engineering

\& Sciences Publication

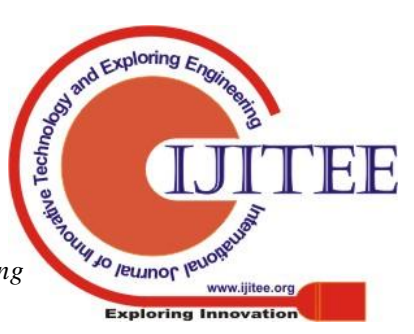




\section{A. Measurement of density:}

Estimation of thickness is done by IR sensors which are balanced aside of each road. Two IR sensors are used for each road to pick traffic condition on each road. For this, road is withdrawn into three locales from the traffic sign to pick low, medium and high traffic level on road. Each district is a level of low, medium or high traffic level to which express pre-portrayed time between times are ought to be depended in order to achieve time profitable traffic the experts. The sensors are mounted around the beginning of second and third zones which are the degrees of medium and high traffic levels deliberately.

\section{Low traffic thickness:}

To show low traffic level no sensor is used; in light of the way that, no yield from sensor to microcontroller is standard as low traffic level on road and least pre-portrayed time is doled out to that road of which no sensor is giving yield.

(Note: No sensor is used for this case imagining surprisingly populated region of progress. In any case, a sensor can be used for this zone if region of progress has low masses where dodging the green sign for any road is conceivable)

\section{Medium traffic thickness:}

Medium traffic level is shown using a sensor mounted close to the beginning of a resulting space. Right when this sensor gives yield, it endorses that traffic on the given road is in a medium range. So time between time, predefined for an occasion of medium thickness traffic is based on this road when sign turns green.

\section{High traffic thickness:}

Estimation of high traffic level is done by using two sensors mounted on each road. Right when both the sensors on a road gives yield it embraces that traffic on the given road is in the high traffic level range. Consequently, extra time between time is alloted to the road having high traffic thickness to clear traffic on it.

\section{B. Dissipating of time:}

Dissipating of time is done everything considered that for each traffic level condition like low, medium and high, enough time breaks get conveyed for a particular road having any three of the conditions. With the objective that none of the road gets extra chance to clear its traffic nor gets less time. The microcontroller picks decision about undertaking of time to a particular road in consent to the yield of sensors on that road just before the minute the sign for given road turns green. For a road having low thickness traffic that is no sensor on that road was moving yield going before the minute when sign makes strides toward normal propriety to that road, least time is doled out when sign turns green. With the objective that no extra time is given to the road under idea, keeping up a key separation from insignificant holding up by the traffic on a road whose sign should turn green straightaway. for instance $5 \mathrm{sec}$. for low traffic thickness. (Note: If a sensor is used to show low traffic thickness other than and it isn't giving any yield, we can keep up a key partition from the green light time for that particular road or everything considered if giving yield we can enjoy a reprieve as explained).For a road having medium thickness traffic that is first sensor which is mounted close to the beginning of second segment was moving yield going before the minute when sign breezes up being earth reasonable to that road, such a lot of time between time is doled out to that road as to allow enough time zone for the traffic on that road to be cleared when sign turns green. This keeps up a vital division from silly holding up by the traffic on the going with road what's beginning and end the furthermore getting worthy chance to clear traffic on the present masterminding road. for instance 10 sec. for medium traffic thickness. For a road having high thickness traffic that is both the sensors offering yield going before the minute when sign breezes up being earth sensible to that road, most insane time between time is facilitated to this road to clear traffic on it effectively. for instance $20 \mathrm{sec}$. for high traffic thickness. Absolutely when disintegration sign occurs for any road, structure closes down its average endeavor and starts executing the road which is to be supplant. For this condition, rich time say $15 \mathrm{sec}$. is given to that road so any emergency vehicle on a road can pass the sign without contributing such a lot of centrality at it.

\section{Emergency repeal:}

The emergency annul is done by using a ton of RF transmitter and Receiver. RF encoder is used to submit gathered RF accentuate for each road. RF decoder is used to see the RF sign repeat and choose to which road it has a spot.

Accurately when RF transmitter sends unequivocal discontinuous sign alloted to a specific street through encoder, at the decoder end it decides to which street it has a spot and sends the relating information to microcontroller. By then microcontroller makes green light $\mathrm{ON}$ for that specific street to which transmitted sign rehash has a spot not long after fitting a little while later preparing street. After the green light composed out hops out at a street which had been supersede, ordinary framework undertaking resumes with turning ON the green light for the street which is near a street which had been under regulating just before renounce sign has happened. The crisis vehicle passing on $\mathrm{RF}$ transmitter can transmit the denial signal if there ought to be an event of crisis. To make essential for the authority to know unequivocally which street he needs to remove, principal names/pictures are given to ways at each traffic sign social affair and transmitter contraption additionally has same names/pictures on it to demonstrate which rehash has a spot with which street. So administrator can deny express street by sending rehash of a street he needs to supersede. The standard names/pictures ought to be offered to boulevards at each sign intermingling point in such a structure, that a name/picture on a transmitting contraption and of a street at each intermixing have a spot with same rehash. 


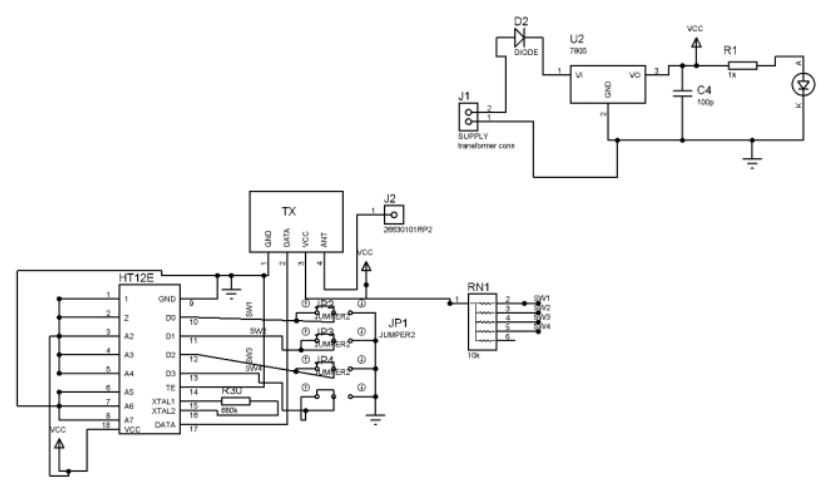

Transmitter section: Transmitter takes after a LED. This IR transmitter dependably passes on IR shafts from it. The working voltage of this IR transmitter is 2 to $3 \mathrm{v}$. These IR (infra red) shafts are ambiguous to the human eye.Butwecanview these IR bars through camera.

\section{Receiver section:}

IR beneficiary gets IR shafts that are transmitted by IR transmitter. Reliably IR ace has high confinement arranged by super ohms, when it is getting IR bars the check is low. The working voltage of IR beneficiary other than 2 to $3 \mathrm{~V}$.

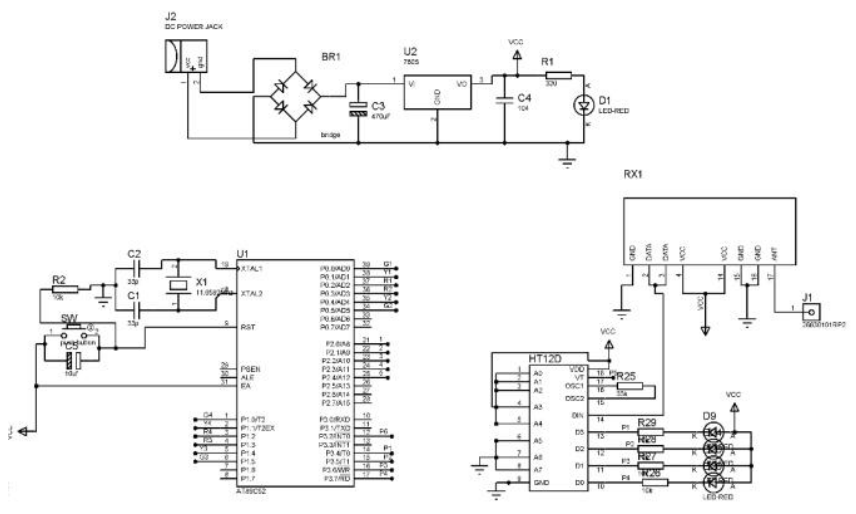

Schematic diagram:

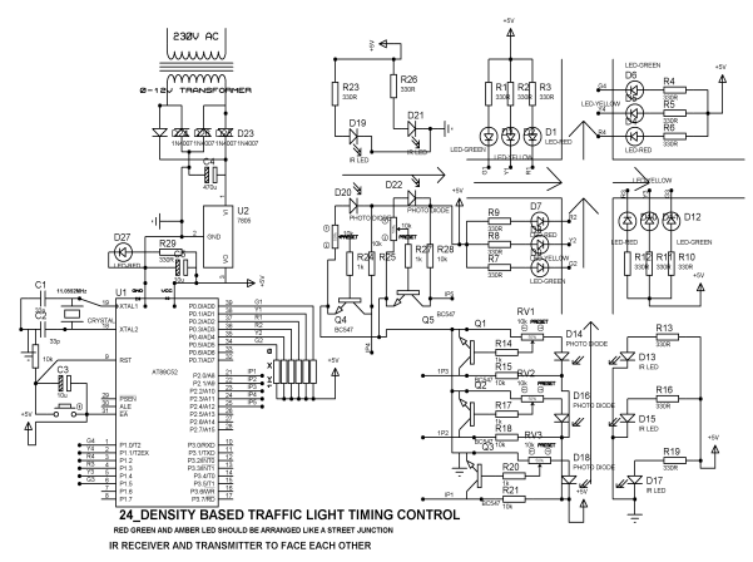

IV. WORKING:

The undertaking utilizes the IR interference thought for giving technique for reasoning state change to the commitment of the MC as clarified already. The undertaking utilizes undeniable IR diodes facings photodiodes. Thusly the transistors Q1, Q2, Q3, Q4, Q5 are in driving state. As the expert of those transistors are related with isolating port pins structure as a commitment in regards to the program to the executed subject to change of reason state.

Twelve number of LEDs tending to as sign lights are related with the yield of the MC in sink mode to port 'o', port 1 and port 2. While the majority of the information beginning from Q1 to Q5 are in defend low express, the yield LED's i.e., 3 for each intersection point that is Red, Amber and Green of each side procedure for a four traffic get together quest for after switch ON green masterminding in fixed breaks a dynamic clockwise way. Thusly during low traffic thickness in one of the way, fixed green making game-arrangements for every path in an intersection point are given. While any of the way is demolished with more no.of vehicles the IR blocking occurs. There are three zones with three arrangement of IR seeing framework In this undertaking the transistors Q1 to Q5 goes high by significance of IR counteractive action while the vehicle comes in the midst of the photodiode and the IR diode.

This, the assistance high obvious at the MC information changes the green $\mathrm{ON}$ time to a higher shimmer for interfacing more vehicles to encounter. After in the long run correspondingly some other way gets more traffic, the dynamic arranging gets thusly associated for that way. Every way is withdrawn into 3 dynamic zones, each zone watching out for some particular length. In light of the IR impedance the green $\mathrm{ON}$ time makes, in that limit more the vehicle longer will be the green sign time. Thusly unfathomable time control is made subject to the traffic thickness. green ON time stretches out, thusly more the vehicle longer will be the green sign time. Along these lines stunning time control is exhausted subject to the traffic thickness.

\section{RESULT:}

By understanding the proposed structure, we can defeat over the top streets turned stopping regions hence inciting smooth traffic stream. It gives a modernized system for controlling traffic. This is an anticipated undertaking which should be possible on an enormous scale. The diagram depicts the traffic thickness at the affiliations, amassed unendingly of the year. This paper plots the traffic seeing which is basic in the decrease of traffic blockage.

\section{INCREASEOF TIME DUE TO DENSITY PARTICULAR LEVEL:}

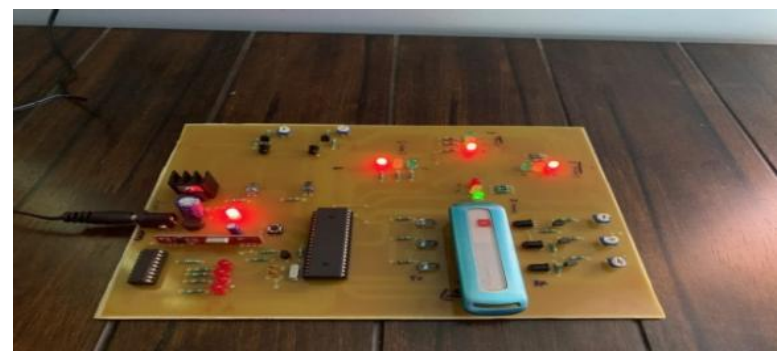


In the above framework we can see that increaseof time delay in level-2. It is a deferred result of development of thickness.

Here we set an article in level-2, with the target that the specific IR sensors isolates the closeness of thing. In this instrument the IR transmitter, transmits the sign to recipient. On the off chance that there is no vehicle among transmitter and gatherer there is no time delay for that level. On the off chance that there is a vehicle between them the time delay for that specific level gets increments. In light of these fragment the time delay at level-2 increments, with the target that relating green drove shines additional time wound from different levels.

\section{REMOTE OVERRIDE IN AN EMERGENCY:}

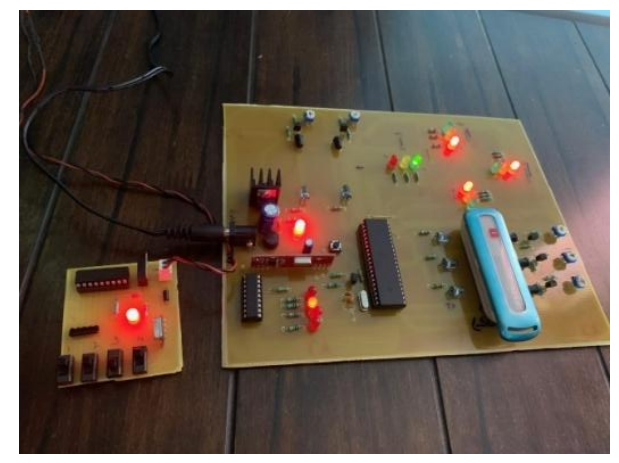

By sensibility of crisis two or three vehicles requires a surprising access to move their vehicles. In that time by utilizing remote refutation oblige we can without a gigantic measure of a stretch control the sign. A couple of vehicles like ambulances, police vehicles they requires awesome access in that time in the event that they have radio irregular sign remote they can without a lot of a stretch control the sign.

In the organization we can see that a level-3 get in the remote gets squashed. With the target that relating level allocates sign and green drove shines. The green drove presentations green sign until the vehicle crosses the junction.After vehicle crosses the blend the traffic signal again works subject to the thickness.

\section{CONCLUSIONS:}

The proposed framework is a thickness based traffic divulgence structure that gives capable traffic light control by convincing senseless pay amazing character to street to an enormous degree. The extent of IR sensors showed will pick the thickness level of traffic and subsequently it fills in as a need based structure as it gives crisis evacuate highlights.

\section{FUTURE EXPANSION:}

The future level of this undertaking lies in the amazing usage of a moving control framework by improving the accuracy of the structure. Execution of the proposed framework in gigantic scale yields extraordinary conditions like less stop up and consequently less utilization of fuel.
Notwithstanding the manner by which that the model worked all around enough with basic yields, the honest to goodness circumstance will be a lot other than testing and referencing. Maybe a few the difficulties that ought to be considered are recorded as looks for after

- Low range IR sensors may not be a response for long range hailing framework. We may depend on ultrasound or radar structures for enormous scale set-ups.

- Next is the impact of stray flag that may change the looking sensor receptors and lead to passing on false data to the microcontroller.

- Periodic checking of the precision and exactness is a level out need for adequate activity of this model.

\section{Prospering first:}

It must be completely ensured that no trade off is being made on prospering issues, for example an optional remain by set-up that can change over from automated to manual mode, ought to be given if there should be an occasion of sensor or circuit breakdowns with the target that vehicular get-together does not leave hand. As a crucial piece of future progressions, the traffic check post might be related by remote transmitters by which the intersection point focuses ahead might be a hurting for the traffic that is drawing nearer. This might be cleaned the interfacing the sensor arrange with GPS structure and short wave radio transmission signals. This will go about as a feed forwardsystemmakingthe hailing systemeven capably smooth and blockage free.

\section{REFERENCES}

1. A. Siddique, WB: Dhaka's average traffic speed $7 \mathrm{kmph}$. [online] Dhaka Tribune. Available at:, Jul. 2017, [online] Available:

http://www.dhakatribune.com/bangladesh/dhaka/2017/07/19/d haka-average-traffic-speed-7-kmph/.

2. A. Jadhav, B. Madhuri, T. Ketan, "Intelligent traffic light control system (ITLCS)", Proceedings of the 4th IRF international conference, 16 March 2014.

3. Mohammad ShahabUddinAyon Kumar Das, Md. Abu Taleb, -Real-time Area Based Traffic Density Estimation by Image Processing for Traffic Signal Control System: Bangladesh Perspectivell IEEE 21-23 May 2015.

4. Bilal

Ghazal,KhaledEIKhatib,KhaledChahine,MohamadKherfan, —Smart Traffic Light Control System IEEE 2016.

5. M. AshwinKumaar, G. Akshay Kumar, S.M. Shyni -Advanced Traffic Light Control System Using Barrier Gate andGSM , 2016 International Conference on Computation of Power, Energy Information and Communication (ICCPEIC).

6. GundeepKaur, Sonia Sharma," Traffic Management Using Digital Image Processing", IJCST Vol. 8, ISSue 2, Aprll June 2017.

7. Md. MunirHasan, AminulHoque, GobindaSaha, and Md. BadruddojaMajumder. "Smart Traffic Control System with Application of Image Processing Techniques", 3rd International Conference on Informatics, Electronics \& Vision 2014.

8. Anil Vishwasrao, OmkarRamdasGaikwad, Prof. KanchanPujari, TejasTalathi, "Image Processing Based Traffic Light Control", International Journal of Science, Engineering and Technology Research 


\section{ABOUT AUTHORS:}

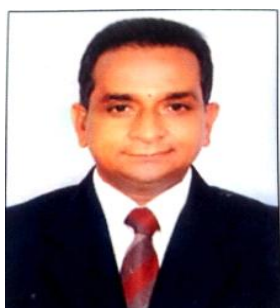

\section{Dr. P. V. RAMA RAJU}

Presently working as a Professor and HOD of Department of Electronics and Communication Engineering, S. R. K. R. Engineering College, AP, India. His research interests include Biomedical-Signal Processing, Signal Processing, Image Processing, VLSI Design, Antennas and Microwave Anechoic Chambers Design. He is author of several research studies published in national and international journals and conference proceedings

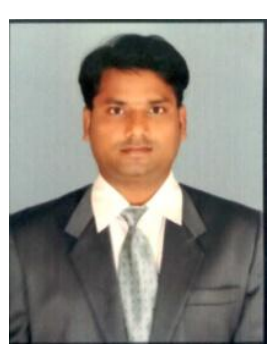

\section{G. NAGA RAJU}

Presently working as assistant professor in Dept. of ECE, S.R.K.R.Engineering College, Bhimavaram, AP, India. He received B.Tech degree from S.R.K.R Engineering College, Bhimavaraman in 2002 and M.Tech degree in computer electronics specialization from Govt. College of Engg., Pune University in 2004. His current research interests include Image processing, digital security systems, Signal processing, Biomedical Signal processing, and VLSI Design

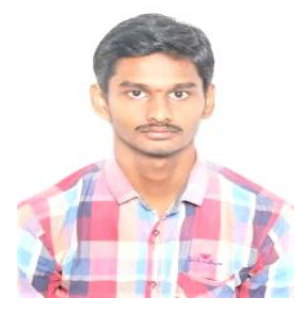

\section{N. GANESWAR REDDY}

Presently pursuing Bachelor of Engineering degree in Electronics \& Communication engineering at S.R.K.R. Engineering College, AP, India

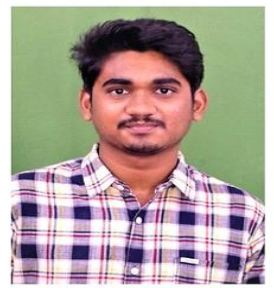

\section{SAI KANNA}

Presently pursuing Bachelor of Engineering degree in Electronics \& Communication engineering at S.R.K.R. Engineering College, AP, India

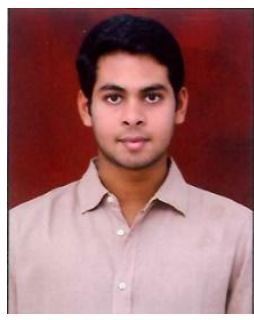

\section{B. LAKSHMANA SASHANK}

Presently pursuing Bachelor of Engineering degree in Electronics \& Communication engineering at S.R.K.R. Engineering College, AP, India 